August 2, 2018

\title{
Comparison of numerical methods for the calculation of cold atom collisions.
}

\author{
G. H. Rawitscher ${ }^{a}$, B. D. Esry ${ }^{b}$, E. Tiesinga ${ }^{c}$, J. P. Burke, Jr ${ }^{d}$, I. Koltracht ${ }^{e}$ \\ ${ }^{a}$ Physics Department, Univ. of Connecticut, Storrs, CT, 06268; \\ ${ }^{b}$ ITAMP,Harvard Smithonian Center for Astrophysics, Cambridge, MA 02138; \\ ${ }^{c}$ Atomic Physics Division, National Institute of Standards and \\ Technology,Gaithersburg, MD 20899. Permanent Address, Department of \\ Chemistry and Biochemistry, U. Maryland, College Park, MD 20742; \\ ${ }^{d}$ Department of Physics and JILA, Univ. of Colorado, Boulder, CO \\ 80309-0440; \\ ${ }^{e}$ Department of Mathematics, Univ. of Connecticut, Storrs, CT 06268
}

\begin{abstract}
Comparison between three different numerical techniques for solving a coupled channel Schrödinger equation is presented. The benchmark equation, which describes the collision between two ultracold atoms, consists of two channels, each containing the same diagonal Lennard-Jones potential, one of positive and the other of negative energy. The coupling potential is of an exponential form. The methods are i) a recently developed spectral type integral equation method based on Chebyshev expansions, ii) a finite element expansion, and iii) a combination of an improved Numerov finite difference method and a Gordon method. The computing time and the accuracy of the resulting phase shift is found to be comparable for methods i) and ii), achieving an accuracy of ten significant figures with a double precision calculation. Method iii) achieves seven significant figures. The scattering length and effective range are also obtained.
\end{abstract}




\section{Introduction}

The collision between two ground state atoms at low $(\mu \mathrm{K})$ temperature poses challenging computational problems which can be summarized by the words "long range" and "coupled channels". The former problem arises from the fact that in the collision process the atomic clouds "polarize" each other, leading to long ranged dispersion potentials. 1 The latter problem arises from the fact that the internal hyperfine structure of the atoms leads to a set of coupled Schrödinger equations that describe the transitions which an atom in the incident channel can make to many of these hyperfine states. Accurate calculations of the scattering properties and wave functions of the atom-atom collision are crucial, since the macroscopic shape of the Bose-Einstein condensate as well as the lineshapes of the photoassociation spectral depend sensitively on these quantities .

The lower the incident energy of the atoms, the larger are the distances for which the potentials affect the phase shifts. This can be seen from the WKB approximation to the phase shift which contains integrals over the local wave length $k(x)=[2 \mu(E-V(x))]^{1 / 2}$ of the form

$$
\int k(x) d x \simeq \int k_{0} d x-\frac{1}{2 k_{0}} \int V(x) d x,
$$

since the first significant term in the expansion above contains the ratio of the potential to the asymptotic wave number. Further, when negative energy channels are coupled to the positive energy incident channel, it can become difficult to enforce the appropriate decaying wave function boundary condition, with resulting loss of stability, depending on the algorithm used.

There are various calculational methods available for dealing with this scattering problem: modified Numerov, Gordon's, 1 a finite element method (FEM), and a recently developed method that consist of replacing the coupled differential equations by equivalent integral equations (IEM) 1.0 In addition, there are more sophisticated finite difference methods $\$$; we, however, will not consider these methods here since they are not as widely in use. Methods involving the representation of a continuous function by a finite set of sampling points haye been discussed 9 One such method led to the mapped Fourier grid method 10 which has been employed for the calculation of the collision between cold atoms.11 The interaction of cold atoms with surfaces has also been discussed 12 including how to implement boundary conditions..

Depending on the degree of accuracy required and the ease of performing the calculation, any one of these methods may be the most suitable for a particular situation. It is nevertheless of interest to compare these methods with each other as far as accuracy, stability and numerical complexity are concerned. It is the purpose of this paper to compare the three methods, by numerically evaluating a benchmark test case described below. 
"Numerical computational stability" is a many-faceted concept. It manifests itself through the degree of accuracy obtained. There are at least three ingredients:

- i) How the numerical truncation error of the algorithm is offset by the presence of the accumulation of round-off errors. The larger the number of mesh points the smaller the truncation error, but the larger is the corresponding overall round-off error. Different algorithms strike a different balance between these two errors.

- ii) The sensitivity of the final result to the errors in the input data, such as the potentials, masses, etc. This sensitivity is expresses by the "condition" of the formulation model.

- iii) How the asymptotic boundary conditions are achieved, both in the open and the closed channels. For the latter, the growing solutions that contaminate the decaying solutions have to be eliminated. Each algorithm proceeds by a different method. For example, in the IEM we introduce scaling factors in each partition that prevent the unwanted solution. In the finite difference methods, one has to integrate inward and outward and then match at some intermediary distance. Here the matching matrix can introduce errors. With the finite elements, the closed channel solutions are forced to be zero at the final matching point, automatically eliminating the unwanted growing solutions.

It is not the purpose of this paper to investigate in detail the stability properties of the three algorithms described in the present study. For that, a detailed comparison of the numerical solution with an exact solution for an artificial test case would have to be performed. Rather, we here attempt to obtain some numerical evidence for the degree of stability of the three methods for a realistic example.

\section{The Test Case}

The test case we have chosen consists of a model calculation that captures the essence of the collision of two ultra-cold ${ }^{2} \mathrm{~S}$ alkali atoms. The characteristic feature of such collisions is that in going from large to small internuclear separations a change of coupling schemes occurs. Asymptotically, the hyperfine structure of the individual alkali atoms dominates, while at short internuclear separations the molecular $X^{1} \Sigma_{g}$ and $a^{3} \Sigma_{u}$ potentials dominate. A basic understanding of the properties of these two Born-Oppenheimer potential curves can be found in any text book on quantum mechanics that discusses the electronic 
structure of a $\mathrm{H}_{2}$ molecule. Here it is sufficient to realize that they have an identical long-range attractive van der Waals behavior and are split exponentially via an exchange mechanism at shorter internuclear separations.

The simplest multi-channel potential that captures this physics is thus a two channel model. Taking into account that for small collision energies the nuclear rotation can be safely ignored, the Hamiltonian is conveniently parametrized as

$$
\left\{-\frac{\hbar^{2}}{2 \mu} \mathbf{1} \frac{d^{2}}{d r^{2}}+\left(\begin{array}{cc}
V_{\mathrm{LJ}}(r) & A e^{-b r} \\
A e^{-b r} & V_{\mathrm{LJ}}(r)+E_{\mathrm{hf}}
\end{array}\right)-\mathbf{1} \mathcal{E}\right\}\left(\begin{array}{c}
\psi_{P} \\
\psi_{N}
\end{array}\right)=0
$$

where the reduced mass $\mu=M / 2, E_{\mathrm{hf}}$ is the asymptotic splitting between the two channels, and $\mathcal{E}$ is the total energy in the system. The $r$-dependent potentials of our test problem are the Lennard-Jones potential $V_{\mathrm{LJ}}(r)=C_{12} / r^{12}-$ $C_{6} / r^{6}$ and an off-diagonal exchange coupling given by $A e^{-b r}$. The functions $\psi_{P}$ and $\psi_{N}$ describe the wavefunction for the open and closed channel, respectively. Notice that the zero of energy is located at the lowest of the two asymptotes.

Obviously this Hamiltonian is set up in terms of the atomic basis. At large internuclear separation the Hamiltonian reduces to a diagonal matrix. In fact, the Hamiltonian would be diagonal for all internuclear separation if the exponential off-diagonal potential were absent. It turns out that for internuclear separations where this term is large compared to $E_{\mathrm{hf}}$ it is informative to calculate the adiabatic potentials by diagonalizing the potential term of the Hamiltonian at each internuclear separation. At shorter distances the resulting potentials correspond to a very deep $\mathrm{X}^{1} \Sigma_{g}$ and a shallow $a \Sigma_{u}$ potential, to a good approximation.

For two colliding ultra-cold ${ }^{2} \mathrm{~S}$ Na atoms,realistic values of the constants are $M=22.9897680 \mathrm{amu}, C_{6}=1472$ a.u. $\left(a_{0}\right)^{6} 13 C_{12}=38 \times 10^{6}$ a.u. $\left(a_{0}\right)^{12}, \mathrm{~A}=2.9$ a.u., b=0.81173 $a_{0}$, and $E_{\mathrm{hf}}=0.2693 \cdot 10^{-6}$ a.u. This choice of $E_{\mathrm{hf}}$ is approximately equal to the atomic hyperfine splitting of the ${ }^{2} S \mathrm{Na}$ atom. The total energy $E=3.1668293 \times 10^{-12} a . u$. corresponds to a temperature of $1 \mu \mathrm{K}$. Since $E \ll E_{\mathrm{hf}}$, the energy in the second channel is negative, i.e., only one of the two channels is asymptotically accessible. In the above $a . u$. stands for atomic units, and $a_{0}$ is the Bohr radius.

The conversion into entirely $a_{0}$ units is achieved by dividing the above equation by $2 \mu / \hbar^{2}$. One obtains

$$
\left(-\frac{d^{2}}{d r^{2}}+\mathcal{V}-\mathcal{E}\right)\left(\begin{array}{c}
\psi_{P} \\
\psi_{N}
\end{array}\right)=0
$$

where $r$ is in units of $a_{0}$ and the potential and energy matrices, $\mathcal{V}$ and $\mathcal{E}$ respectively, are in units of $\left(a_{0}\right)^{-2}$. The conversion of a quantity in a.u. units to $\left(a_{0}\right)^{-2}$ units is achieved by multiplying the former by $\mu=22.989768 \times$ $1822.888506(\text { a.u. })^{-1}\left(a_{0}\right)^{-2}$. The potential matrix is

$$
\mathcal{V}=\left[\begin{array}{cc}
\tilde{V} & \tilde{U} \\
\tilde{U} & \tilde{V}
\end{array}\right]
$$


where $\tilde{V}=V \times \mu, U=\tilde{U} \times \mu$, and the energy matrix is

$$
\mathcal{E}=\left[\begin{array}{ll}
k^{2} & \\
& -\kappa^{2}
\end{array}\right] \text {. }
$$

Here $k$ and $\kappa$, the wave numbers in each channel, are given by $k=\sqrt{E \times \mu}$ and $\kappa=\sqrt{E_{h f} \times \mu-k^{2}}$. In our numerical example, the corresponding values are $k=3.643004224146145 \times 10^{-4}\left(a_{0}\right)^{-1}$ and $\kappa=0.1062338621818394\left(a_{0}\right)^{-1}$. The wave function is normalized so that asymptotically it becomes

$$
\left(\begin{array}{l}
\psi_{P} \\
\psi_{N}
\end{array}\right) \approx\left(\begin{array}{l}
\sin (k r)+K_{1} \cos (k r) \\
K_{2} \exp (-\kappa r)
\end{array}\right)
$$

where $K_{1}$ and $K_{2}$ are two elements related to the real scattering $R$ matrix, in terms of which the phase shifts can be obtained.

In this model, the diagonal potential extends to considerably larger distances than the coupling potential. Further, between 6 and $10 a_{0}$ the diagonal potential is very deep leading to many oscillations in the wave functions. For example, near $5.5 a_{0}$ the local wave length $\lambda$ in both channels is $\simeq 0.25 a_{0}$, near $8.5 a_{0}$ $\lambda \simeq 1.2 a_{0}$, with smaller ripples superimposed, and near $20 a_{0}$ the local wave length has increased to $\simeq 4 a_{0}$ At distances less than $4 a_{0}$ the repulsive portion of the potential becomes very large making the wave function very small. In order to allow for the singularity of the diagonal potentials near the origin, a parameter $R_{\text {cut }}$ is defined, and the wave functions are set to zero in the interval $\left[0, R_{\text {cut }}\right]$. A value of $R_{\text {cut }}=4.0 a_{0}$ is found to be satisfactory. In addition, the calculation is carried out to a maximum radius, $R_{\max }$, beyond which all potentials are set equal to zero. In our calculations, $R_{\max }$ is set equal to $500 a_{o}$. When $R_{\max }$ is increased further, the values of $K_{1}$ and $K_{2}$ still change beyond the 6 th. significant figure, as can be seen from the Table in Appendix, even though the Lennard-Jones potential is less than $-3.95 \times 10^{-9} a_{0}^{-2}$. The large effect on the phase-shift produced by such a small potential is due to the occurrence of the factor $1 / k \simeq$ $2.75 \times 10^{3}$ in the integrals involving the potential tail, as was already pointed out in the introduction. Rather than numerically calculating such changes, it is preferable to employ perturbation methods, which are described in Appendix 2.

\section{A. The Integral Equation Method.}

In this method the differential equation $\left(\frac{d^{2}}{d r^{2}}+\mathcal{E}\right) \psi=\mathcal{V} \psi$ is transformed into the Lippmann-Schwinger integral equation

$$
\psi(r)=F(r)+\int_{0}^{R_{\max }} \mathcal{G}_{0}\left(r, r^{\prime}\right) \mathcal{V}\left(r^{\prime}\right) d r^{\prime}
$$

where $F(r)$ is a undisorted wave function, like $(\sin (k r), 0)$, and $\mathcal{G}_{0}\left(r, r^{\prime}\right)$ is the undistorted Green's function matrix. A motivation for such an approach is 
that the solutions of integral equations have better numerical stability than the solutions of differential equations. One common objection to the use of integral equations has been that the solution leads to large matrices which are not sparse and hence require substantially larger amounts of computing time than the sparse matrices of differential equations. This objection was overcome in our integral equation method (IEM) by dividing the whole radial interval into partitions. The integral equations in each partition lead to dense matrices of small dimension, but the matrix that combines the local solutions into the global one, albeit of large dimension, is sparse. It should be noted that this latter property is valid only in configuration space, because only in this space do the Green's functions have the required semi-separable nature. In the present version of the IEM method the (variable) size of each of the partitions is determined in terms of two parameters $N L$ and $\epsilon$ as follows. In each radial region a local wave length in channels 1 and 2 is obtained as $2 \pi / \sqrt{\left|k^{2}-\tilde{V}(r)\right|}$, and $2 \pi / \sqrt{\left|-\kappa^{2}-\tilde{V}(r)\right|}$. The smaller of the two local wavelengths is taken, and the size of the partition in that region is determined such that there are a given total number $N L$ of Chebyshev points per local wave length. Allowing for the fact that in each partition there are 16 Chebyshev points, the average length of a partition for a given local wave length $\lambda$ is $\lambda \times 16 / N L$. The length of each partition is subsequently readjusted using the tolerance parameter $\epsilon$ as follows. According to the IEM method, 1 in each partition two sets of "local" functions are calculated in terms of which the global function $\psi$ is obtained as a linear combination. The accuracy of each of the local functions can be determined by the size of the coefficients of the highest order Chebyshev polynomials. If the relative accuracy of the local functions in a given partition is larger than $\epsilon$, then that partition is divided in half, and the testing is continued. If the initially chosen value of $N L$ is too small, then the initial partitions are too large, and many of the partitions are subsequently reduced by the $\epsilon$ criterion. In this case the final number of partitions $M$ becomes larger than their initial value. If the chosen value of $N L$ is too large, then most of the partitions are unnecessarily small, and the value of $M$ is too large, leading to a larger accumulation of roundoff errors for the final elements of the $K$-matrix. An exception is the interval $\left[0, R_{c u t}\right]$. This interval is considered as one partition, containing a total of 16 Chebyshev points. This is sufficient since the wave function is very small in this region (less than the desired accuracy for the values of $K$ ), and the values of $K$ were found to be stable to 11 significant figures as $R_{c u t}$ was varied below $4.0 a_{0}$.

In summary, for a given value of $\epsilon$, the value of $N L$ was varied until the smallest number of partitions $M$ was obtained. An example is given in the table below.

Table 1. Values of $K_{1}$ and number of partitions $M$ as a function of NL for the tolerance $\epsilon$. $=10^{-9}$ 


\begin{tabular}{|c|c|c|c|}
\hline$N L$ & $M$ & $K_{1}$ & $K_{2}$ \\
\hline 10 & 150 & -0.31233398338809 & 6.5761303971514 \\
\hline 20 & 153 & -0.31233398339572 & 6.5761303973071 \\
\hline 30 & 144 & -0.31233398339229 & 6.5761303972290 \\
\hline 40 & 154 & -0.31233398339070 & 6.5761303972039 \\
\hline 50 & 177 & -0.31233398338870 & 6.5761303971639 \\
\hline
\end{tabular}

From this table one can find a value of $K_{1}=-0.3123339834$ and $K_{2}=$ 6.576130397 which are stable to ten significant figures. For values of the tolerance $\epsilon$ between $10^{-13}$ and $10^{-3}$ a good compromise value of 10 for $N L$ was found. The corresponding values of $M$ and the corresponding accuracy of $K_{1}$ are listed in Table 2 for several values of $\epsilon$.

Table 2. Accuracy for $K_{1}$ and number of partitions $M$ for a given value of the Tolerance $\epsilon$, with $N L=10$

\begin{tabular}{|c|c|c|c|}
\hline$\epsilon$ & $M$ & $K_{1}$ & \# of Sign. Figs. \\
\hline $10^{-2}$ & 28 & $\mathbf{- 0 . 3 1 2 4 3 3 3 7 4 0 2 0 9 9}$ & 3 \\
\hline $10^{-3}$ & 30 & $\mathbf{- 0 . 3 1 2 3 3} 467247746$ & 5 \\
\hline $10^{-5}$ & 67 & $\mathbf{- 0 . 3 1 2 3 3 3 9 8} 457315$ & 8 \\
\hline $10^{-7}$ & 106 & $\mathbf{- 0 . 3 1 2 3 3 3 9 8 3 7 0 6 3 7}$ & 8 \\
\hline $10^{-9}$ & 158 & $\mathbf{- 0 . 3 1 2 3 3 3 9 8 3 3} 8809$ & 10 \\
\hline $10^{-11}$ & 214 & $\mathbf{- 0 . 3 1 2 3 3 3 9 8 3 3} 8869$ & 10 \\
\hline $10^{-13}$ & 574 & $\mathbf{- 0 . 3 1 2 3 3 3 9 8 3 3 3} 8534$ & 10 \\
\hline
\end{tabular}

From this table it appears that beyond $\epsilon=10^{-9}$ the accumulation of roundoff errors begins to dominate, and -0.31233398339 is the best value of $K_{1}$. The distribution of partitions for three tolerance parameters is shown in Fig. 1.The increasingly large spacing of the partitions at the large distances is clear from the figure. In the vicinity of $R \simeq 50$ the density of partitions is high because the negative energy channel has a turning point there. This shows that the local wave-length criterion alone would have been insufficient to determine the partition size.

\section{Scattering Length and Effective Range.}

As a further test of the stability of the IEM method, the Scattering Length $a$ and Effective Range $r_{e}$ are investigated. They are obtained in the limit of small wave number $\mathrm{k}$ from the expression

$$
k / K_{1}=-\frac{1}{a}+r_{e} k^{2}+O\left(k^{3}\right) .
$$


Figure 1: Various IEM partition distributions, described in Table 2. The y-axis represents the partition number $i$, and the x-axis shows the lower boundary of partition $i$. The more points in a particular radial interval, the smaller are the lenghts of the partitions in that interval. All three partition distributions started with the same number of mesh points per local wavelength $(\mathrm{NL}=20)$, but were subsequently modified by the $\epsilon$-accuracy criterion, with $\epsilon=10^{-9}, 10^{-6}$, and $10^{-3}$, respectively. The numbers above each curve represent the number of accurate significant figures achieved for the asymptotic constant $K_{1}$ for each value of $\epsilon$. The large concentration of partitions in the vicinity of $50 a_{0}$ reflects the occurrence of a turning point in the negative energy channel near that distance, where accuracy would have been lost had the initial partition distribution been used. 
The left hand side of the above equation is usually written as $k \cot \delta_{0}$, which is equal to $k / K_{1}$. For a given choice of the truncation radius $R_{\max }$ the value of $K_{1}$ is calculated for two different and small values of $k$ and the values of $a$ and $r_{e}$ are obtained from the two values of $k / K_{1}$ in Eq. (6).

However, as mentioned in the introduction, the values of the scattering matrix $K$ depend on the choice of the truncation radius, the more so the smaller the value of $k$, because of the increasingly non-negligible contributions of the potential beyond $R_{\max }$. Hence, in order to obtain a reliable value of the scattering length, it is advantageous to first correct $K_{1}$ for the contributions beyond $R_{\max }$. This can be done quite simply by using first order perturbation theory, as is detailed in Appendix 1, and as will be demonstrated below.

An example of the variation of the $K$ 's with $R_{\max }$, obtained by the IEM, is given in the table below. All results in this section were obtained with NL = 10 , and $\epsilon=10^{-9}$.

Table 3: Dependence of $K_{1}$ and $K_{2}$ on $R_{\max }$.

\begin{tabular}{|c|c|c|c|}
\hline$K_{1}\left(R_{\max }\right)$ & $K_{1}(\infty)$ & $K_{2}\left(R_{\max }\right)$ & $R_{\max }$ \\
\hline-.313705209 & -.312322902025 & 6.62005410 & 250 \\
\hline-.312333983 & -.312323344009 & 6.57613040 & 500 \\
\hline-.312324588 & -.312323343934 & 6.57555968 & 1000 \\
\hline-.312324073 & -.312323343936 & 6.57558157 & 1500 \\
\hline-.312323719 & -.312323343936 & 6.57558741 & 2000 \\
\hline
\end{tabular}

One can see from the table that the value of $K_{1}\left(R_{\max }\right)$ becomes monotonically less negative as $R_{\max }$ increases, while $K_{2}$ first decreases, and then increases for $R_{\max }>1000$. This behavior can be reproduced numerically by means of perturbation theory, described in the appendix. The usefulness of the perturbative correction is also demonstrated by the stability of the column denoted as $K_{1}(\infty)$, which contains the corrected values of $K_{1}\left(R_{\max }\right)$. The table shows that the perturbative correction increases the stability of $K_{1}$ from 5 or 6 significant figures to 11 , yielding $K_{1}(\infty)=-.31232334394$

The stability of the values of $a$ and $r_{e}$ will be described next. The two smallest values of $k$ to be used in Eq. (6) were approximately $0.1152 \times 10^{-4}$ and $0.3643 \times 10^{-6}$, for which the third order term is smaller than the accuracy of the present method. Their values with and without the perturbative correction of $K_{1}$ are shown in the table below.

Table 4: Dependence of $a$ and $r_{e}$ on $R_{\max }$. 


\begin{tabular}{|c|c|c|c|c|}
\hline$a\left(R_{\max }\right)$ & $a(\infty)$ & $r_{e}\left(R_{\max }\right)$ & $r_{e}(\infty)$ & $R_{\max }$ \\
\hline 852.0123407 & 851.9817159134 & 55.08319944 & 55.1051720100 & 500 \\
\hline 851.9849554 & 851.9817157297 & 55.10378197 & 55.1051694472 & 1000 \\
\hline 851.9837969 & 851.9817157354 & 55.10138602 & 55.1051696827 & 1500 \\
\hline 851.9829235 & 851.9817157362 & 55.10046968 & 55.1051693964 & 2000 \\
\hline 851.9824507 & 851.9817157362 & 55.10031183 & 55.1051691577 & 2500 \\
\hline
\end{tabular}

The table shows that the stability of the scattering length is increased from 5 to 11 significant figures by the perturbative correction, yielding $a(\infty)=$ 851.98171574. Likewise, the stability of the effective range is increased from 5 significant figures to 8 , yielding $r_{e}(\infty)=55.105169$.

\section{B. The Finite Element method}

The second method employs the non-iterative eigenchapne 14 variant of the R-matrix method first introduced by Wigner and Eisenbud.15 The eigenchannel R-matrix method solves the Schrödinger equation within a finite reaction volume $\Omega$ of configuration space, subject to constant normal logarithmic derivative boundary conditions on the surface $\Sigma$ of $\Omega$. The collisional properties of the system, typically represented in terms of an $S$-matrix, are easily obtained once the normal logarithmic derivative $b=(\partial \Psi /$ vitialn $) \Psi^{-1}$ is calculated.

One can obtain the following eigenvalue equation for the normal logarithmic derivative $b$ on the surface $2 \underline{4} 4$ :

$$
\underline{\Gamma} \vec{c}=b \underline{\Lambda} \vec{c}
$$

We solve this equation using the finite-element method (FEM) 5 The FEM divides the radial domain into $N$ sectors (or partitions) and within each sector defines a local basis in much the same spirit as the IEM above. The local basis functions, however, are fifth-order Hermite interpolating polynomials rather than Chebyshev polynomials. The Hermite interpolating polynomials $u_{k}\left(x_{n}\right)$, $k=1-6$, are non-zero only in sector $n$. Here, $x_{n}$ is a rescaled variable defined on the interval $[-1,1]$ that is related to the physical internuclear separation $R$ in sector $n$ through an appropriate linear transformation. The six basis functions are defined through the following boundary conditions:

$$
\begin{array}{lll}
u_{k}(-1)=\delta_{1 k} & u_{k}(0)=\delta_{3 k} & u_{k}(1)=\delta_{5 k} \\
u_{k}^{\prime}(-1)=\delta_{2 k} & u_{k}^{\prime}(0)=\delta_{4 k} & u_{k}^{\prime}(1)=\delta_{6 k}
\end{array} .
$$

A multi-component radial wave function can then be represented by the following expansion on the piecewise polynomials $u_{i}(x)$ :

$$
\Psi(R)=\sum_{i \equiv\{m, k, n\}} c_{i} u_{i}\left(x_{n}\right) .
$$


The set $i$ contains the basis function index $k$, the channel index $m$, and the sector index $n$. The coefficients $c_{i}$ are to be determined subject to a continuity constraint on each channel component and its first derivative across sector boundaries. The assembly stage of the calculation is thus performed prior to the main calculation, in contrast to the IEM where it is performed after. In addition, channel boundary conditions can be imposed quite simply by setting the value of the appropriate coefficient. For instance, the closed channel function is forced to be zero on the surface by setting the coefficient of $u_{5}$ in the last sector to zero.

In the finite element representation, the matrix elements of $\underline{\Gamma}$ and $\underline{\Lambda}$ in Eq.(7) are given by

$$
\Gamma_{i j}=2 \mu \int_{-1}^{1} u_{i}\left(x_{n}\right)(E-H) u_{j}\left(x_{n}\right) a_{n} d x_{n}-\delta_{m, m^{\prime}} \delta_{n, N} \delta_{k, 5} \delta_{k^{\prime}, 6} / a_{n}
$$

and

$$
\Lambda_{i j}=\delta_{m, m^{\prime}} \delta_{n, N} \delta_{k, 5} \delta_{k^{\prime}, 5}
$$

respectively, where $a_{n}=\left(R_{n+1}-R_{n}\right) / 2$. Here $i \equiv\{m, k, n\}$ and $j \equiv\left\{m^{\prime}, k^{\prime}, n\right\}$, and $H$ is the Hamiltonian of the system. Because the basis functions are nonzero only within a given sector, the corresponding matrices have roughly a block diagonal structure. Each sector has an associated block which is coupled only to its nearest neighbors through the continuity conditions. Note that the overlap matrix $\underline{\Lambda}$, whose elements are given by surface integrals over the basis functions 14 is particularly simple in the FEM representation. It contains only $m_{o}$ non-zero elements (all equal to one), where $m_{o}$ is simply the number of channels open (i.e., $E>V_{m}$ ) on the surface $\Sigma$. The $\underline{\Gamma}$ matrix is symmetric and, as mentioned, can be constructed in a banded format. The integrals representing the matrix elements of $\underline{\Gamma}$ are also particularly simple in the FEM representation. In fact, except for the integral over the interaction potential, all integrals can be evaluated analytically once and for all before hand, significantly decreasing the CPU time necessary to construct the matrix.

At this stage, we are left with a banded generalized eigensystem to solve that typically has large dimensions but has at most only $m_{o}$ non-zero eigenvalues. Although there are standard linear algebra packages which could solve these equations directly, implementing an efficient, general method would be difficult since the non-zero eigenvalues can range between $-\infty$ and $+\infty$ and all $m_{0}$ of them are needed. It has been shown 14 however, that by partitioning the matrices according to whether the basis functions are non-zero (open $\equiv o$ ) or zero $($ closed $\equiv c)$ on $\Sigma$, i.e. by writing

$$
\left(\begin{array}{ll}
\underline{\Gamma}^{c c} & \Gamma^{c o} \\
\underline{\Gamma}^{o c} & \underline{\Gamma}^{o o}
\end{array}\right)\left(\begin{array}{l}
c^{c} \\
c^{o}
\end{array}\right)=b\left(\begin{array}{cc}
0 & 0 \\
0 & \underline{\Lambda}^{o o}
\end{array}\right)\left(\begin{array}{l}
c^{c} \\
c^{o}
\end{array}\right),
$$

Eq. (7) can be reduced to a small $\left(m_{o} \times m_{o}\right)$ eigensystem

$$
\underline{\Omega}^{o o} \overrightarrow{c^{o}}=b \underline{\Lambda}^{o o} \overrightarrow{c^{o}}
$$


where $\underline{\Omega}^{o o}=\underline{\Gamma}^{o o}-\underline{\Gamma}^{o c}\left(\underline{\Gamma}^{c c}\right)^{-1} \underline{\Gamma}^{c o}$. The main computational burden is thus shifted to constructing $\underline{\Omega}^{o o}$. Since $\underline{\Gamma}^{c c}$ is a large banded matrix in the FEM representation, this is most efficiently accomplished by solving the set of linear equations

$$
\underline{\Gamma}^{c c} \vec{X}=\underline{\Gamma}^{c o} .
$$

The solution $\vec{X}=\left(\underline{\Gamma}^{c c}\right)^{-1} \underline{\Gamma}^{c o}$ thus provides the needed matrix inverse. $\underline{\Gamma}^{c c}$ has dimensions $4 M N \times 4 M N$, but with a half-bandwidth (number of non-zero diagonals above the main diagonal) of only $6 M-1$ resulting from the FEM representation. $\underline{\Gamma}^{c o}$ has dimensions $4 M N \times m_{o}$. We use standard LAPACK 16 routines to solve equations 13 and 14 . The eigenvalues $b$ and eigenvectors $\vec{c}^{b}$ completely specify the linearly independent solutions of the Schrödinger equation on the surface $\Sigma$. This information is generally packaged in terms of a $R$-matrix

$$
R_{m m^{\prime}}=\sum_{\beta} Z_{m \beta} b_{\beta}^{-1} Z_{\beta m^{\prime}}^{-1}
$$

where the columns of $Z$ are given by the eigenvectors $\vec{c}^{D}$. The $S$-matrix is then obtained through simple matrix manipulations involving only the $R$-matrix and the two linearly independent solutions of the asymptotic form of the Schrödinger equation.14

For the two-channel test problem described above, Eq. (14) is a $8 N \times 8 N$ matrix equation with a half-bandwidth of 11 , and only one solution is required since there is only a single open channel, $m_{o}=1$. The present formulation of the R-matrix method focuses the computational effort on finding only the relevant scattering information. Thus, only the coefficient in the open channel $K_{1}$ is obtained. The results are shown in the table below as a function of the number of sectors $N$ used.

Table 5. Results for the Finite Element Method

\begin{tabular}{|c|c|}
\hline$K_{1}$ & $N$ \\
\hline \hline-0.312345739663914 & 400 \\
-0.312334009008278 & 800 \\
-0.312334008856244 & 1600 \\
-0.312334008759115 & 3200 \\
-0.312334006921697 & 6400 \\
\hline
\end{tabular}

\section{The Gordon Algorithm.}

The Gordon algorithm, 四 is a well-established numerical method to solve for the scattering solutions of a set of $N_{c}$ coupled radial Schrödinger equations. Similar to the FEM discussed in the previous section, this algorithm is local in 
the sense that the wavefunction is propagated from $R$ to $R+d R$ using only the wavefunction at $R$. The version of the Gordon method used here is described in Ref..t The main difference from the original Gordon method is in replacing the Airy functions, which correspond to the reference solutions for linearized potentials, by $\sin q r$ or $\cos q r$ in each interval $d R$. Here the quantities $q$ are the local wavenumbers of each channel in the interval $d R$. They are obtained by replacing the potential matrix in interval $d R$ by an averaged constant potential, and then diagonalizing this potential. If the local energy is negative then $\exp (q r)$ or $\exp (-q r)$ are used instead. The wave function in the diagonalized interval $d R$ is represented in each channel $n$ by $\Psi_{n}=A_{n}(r) \sin q_{n} r+B_{n}(r) \cos q_{n} r$, and the vector of the coefficients $A$ and $B$ are calculated by solving a system of first order coupled differential equations involving the difference between the true and the averaged potential matrix in interval $d R$. In the intervals where some of the local energies are negative (i.e., some of the q's are imaginary), the undesired exponentially increasing functions are minimized by a "triangularization" method developed by Gordon. Analytic connection formulas between $R$ and $R+d R$ determine $A$ and $B$, and thus the solution is propagated towards the final matching point. Consequently no large linear system needs to be evaluated in the Gordon algorithm and hence the method is not memory limited.

The physical boundary conditions for the solution are obtained by first calculating $\mathrm{N}_{c}$ linear independent solutions from different initial conditions near the origin, and then constructing appropriate linear combination of these "mathematical" solutions. The stepsize $d R$ is calculated on the fly. After a fixed number of steps (say ten) the percentage change of the $A$ and $B$ coefficients between intervals $n$ and $n+i$, where $i \simeq 10$, is compared to an accuracy criterium. If the variation is too large, the step size is repeatedly halved until the accuracy criterion is met. On the other hand if the coefficients hardly changed the step size is doubled. Consequently, if the potential is well approximated by a constant, large steps are taken. Closed channels are removed from the propagation when the corresponding amplitude of the channel wavefunction has become smaller than a threshold parameter. The influence of these components on the scattering properties of the asymptotically accessible channel is then negligible.

Our results with this algorithm for $R_{c u t}=4 a_{0}$ and $R_{\max }=500 a_{0}$ are summarized in Table 6. The columns describe the fractional change of the $A$ and $B$ coefficients, the number of steps, and $K_{1}$, respectively. It is immediately clear that the Gordon method uses many more steps than the other two numerical methods. As discussed before this is not crucial as it is not necessary to store the wavefunction at every step in order to propagate the wavefunction.

Table 6. Results for the Gordon Method 


\begin{tabular}{|l|l|l|}
\hline Fract'l & No of & \\
\hline Change & steps & $K_{1}$ \\
\hline 0.1000 & 7025 & -0.312348088 \\
\hline 0.0500 & 7632 & -0.312340564 \\
\hline 0.0250 & 8353 & -0.312337479 \\
\hline 0.0100 & 9796 & -0.312335340 \\
\hline 0.0050 & 11402 & -0.312334854 \\
\hline 0.0020 & 14454 & -0.312334070 \\
\hline 0.0010 & 18179 & -0.312334067 \\
\hline 0.0005 & 25502 & -0.312334020 \\
\hline
\end{tabular}

\section{Discussion}

A comparison between the three methods is displayed in Table 7 , which lists the number of mesh points required to achieve a certain accuracy for $K_{1}$.

Table 7. Comparison between three methods.

\begin{tabular}{|l|l|l|l|l|}
\hline & & No of & No of & Total No \\
\hline & $K_{1}$ & partit's & Pts/part. & of Points.. \\
\hline \hline IEM & -0.3123339834 & 153 & 16 & 2,448 \\
\hline FEM & -0.312334009 & 800 & 4 & 3,200 \\
\hline Gordon & -0.3123340 & 25,502 & 1 & 25,502 \\
\hline
\end{tabular}

The finite element (FEM) and the integral equation (IEM) methods are nearly identical in performance. Both can easily adjust the size of the partitions to the local conditions of the potentials; both give stability of at least nine significant figures, and both use approximately the same number of mesh points. Their numerical complexity is also comparable since a CPU-time test shows that both use approximately the same computer time for the case tested here. The IEM and the FEM differ in the 8th significant figure (by $2.6 \times 10^{-8}$ ) for $K_{1}$. The reason for this difference is not known, but could be related to the fact that both the FEM and the Gordon methods have not yet fully converged as the number of partitions is increased, as can be seen from Tables 5 and 6 , and in Figs. 2 and 3. These figures show the value of $K_{1}$, from the fifth significant figure onward, as a function of the total number of mesh points for each of the three methods in progressively larger detail. The somewhat slower convergence for the FEM may be due to the fact that the algorithm for determining the partition size, especially in the vicinity of a turning point, is not as refined for the FEM as for the IEM. 
Figure 2: Comparison of the rate of convergence of the numerical value of $K_{1}$ as a function of the total number of mesh-points $N$. On the vertical axis is plotted the value of $K_{1}$ from the fifth significant figure onwards. This is accomplished by subtracting -0.312300000 from each value of $K_{1}$ and multiplying the result by $10^{4}$. The values of $K_{1}$ are taken from Tables 2,5 , and 6 for the IEM, FEM and Gordon methods. They are represented by solid circles, open circles and squares, respectively 
Figure 3: Same as Fig. 2 with a larger magnification. 
The Gordon method is simpler to implement than the two other ones and gives a respectable accuracy of seven significant figures, albeit at the expense of a much larger number of mesh points, which in turn leads to a larger accumulation of roundoff errors. An improved Numerov method gave only four significant figures $\left(K_{1}=-0.31233(2)\right)$ and is not mentioned further. In the IEM, the boundary conditions are built in automatically via the Green's functions, while in the present form of the FEM the solutions with exponential growth are eliminated by forcing the closed channel component to be zero on the surface $\Sigma$. In a separate study of two coupled equations it is shown that there are situations in which the conventional Numerov method has severe difficulty in obtaining the correct asymptotic boundary condition, while both the IEM and the FEM do not.

It should be clear that the accuracy achieved in this benchmark calculation is not directly relevant for physical applications, since the potentials are usually known only to low accuracy. Instead, the accuracy achieved is to be construed as a measure of the stability of the method, which gives an indication of how well the method is expected to perform under more complex situations, such as when many channels are involved, or when the range of the interaction is excessively large, or at very high energies where many oscillations in the wave functions are present.

In conclusion, all three methods performed well in providing a numerical solution to the coupled channel test case examined here. This case could serve as a benchmark calculation for testing additional methods, since the effective range and the scattering length are also calculated. Further comparison of various methods under more complex conditions would be desirable in order to determine the conditions under which a particular one of the methods would be preferable.

Acknowledgments: E.T. would like to acknowledge useful discussions with Dr. Fred Mies, who has implemented the original version of the Gordon method used by the authors.

\section{Appendix 1. Perturbative treatment of the long-range corrections.}

As the value of $R_{\max }$ (this is the truncation radius beyond which all potentials are set to zero) is increased, the values of $K_{1}$ and $K_{2}$, defined in Eq. (5) change slightly because of the non-zero value of the potential at large distances. In order to extrapolate the $K$ values to $R_{\max }=\infty$, it is preferable to include the long-range tail of the potential perturbatively rather than numerically. Showing 
how this can be done is the purpose of this appendix. It is of course alsopossible to include the long range tails numerically, as has been done in Ref 18 The variation of the $K$ 's with large distances, as given in Table 3, can be reproduced numerically by means of perturbation theory, as will now be shown.

For this section we assume that the radial distance is sufficiently large so that the coupling potential between the two channels is negligible. Thus, only the effect of the diagonal potential in each channel $i$ on $K_{i}$ needs to be considered. We also continue to assume that the angular momentum number $\ell$ is zero. Both assumptions can removed by a generalization of the present discussion.

\section{A. The positive energy channel.}

We will denote two consecutive values of $R_{\max }$ by $T_{1}$ and $T_{2}$., respectively, and assume that the numerical calculation extends out to $T_{1}$. We denote by $V_{1}(r)$ the diagonal potential for $r \leq T_{1}$ and set $V_{1}(r)=0$ for $r>T_{1}$. The corresponding wave function is denoted by $\psi_{1}$ which is equal to the wave function $\psi_{P}$ known numerically. Because $V_{1}(r)=0$ for $r>T_{1}$,

$$
\psi_{1}(r)=\sin (k r)+K_{1}\left(T_{1}\right) \cos (k r) .
$$

Similarly, $V_{2}(r)$ is equal to the diagonal potential for $r \leq T_{2}$ and $V_{2}(r)=0$ for $r>T_{2}$. The corresponding wave function, to be calculated perturbatively, is denoted by $\psi_{2}$. It obeys the equation $\left(d^{2} / d r^{2}-V_{2}+k^{2}\right) \psi_{2}=0$, which can be written

$$
\left(d^{2} / d r^{2}+k^{2}\right) \psi_{2}=V^{\prime} \psi_{2} \quad r \geq T_{1},
$$

where

$$
\begin{array}{lll}
V^{\prime}(r) & =V_{2}(r) \quad \text { for } r \geq T_{1} \\
V^{\prime}(r) & =0 \quad \text { for } r<T_{1} .
\end{array}
$$

Thus, $V^{\prime}$ is the perturbative potential which vanishes outside of the interval $\left[T_{1}, T_{2}\right]$. Using for the inverse of the operator $\left(d^{2} / d r^{2}+k^{2}\right)$ the Green's function

integral expression in terms of $\sin \left(k r_{<}\right) \times \cos \left(k r_{>}\right)$, one obtains the most general form for $\psi_{2}$

$$
\psi_{2}(r)=\alpha \sin (k r)+\beta \cos (k r)+\sin (k r) \Sigma_{c}(r)+\cos (k r) \Sigma_{s}(r)
$$

where

$$
\Sigma_{c}(r)=-\frac{1}{k} \int_{r}^{T_{2}} \cos \left(k r^{\prime}\right) V^{\prime}\left(r^{\prime}\right) \psi_{2}\left(r^{\prime}\right) d r^{\prime}
$$

and

$$
\Sigma_{s}(r)=-\frac{1}{k} \int_{T_{1}}^{r} \sin \left(k r^{\prime}\right) V^{\prime}\left(r^{\prime}\right) \psi_{2}\left(r^{\prime}\right) d r^{\prime}
$$


The coefficients $\alpha$ and $\beta$ are obtained by matching $\psi_{2}$ to $\psi_{1}$ at $r=T_{1}$, i.e., setting the two functions and their derivatives equal. Remembering that $\Sigma_{s}\left(T_{1}\right)=0$, that $(1 / k)\left(d \psi_{2} / d r\right)_{r=T_{1}}=\alpha s_{1}+c_{1}\left(-\beta+\Sigma_{c}\left(T_{1}\right)\right)$, and that $(1 / k)\left(d \psi_{1} / d r\right)_{r=T_{1}}=$ $c_{1}-K_{1}\left(T_{1}\right) s_{1}$, where $s_{1}=\sin \left(k T_{1}\right)$ and $c_{1}=\cos \left(k T_{1}\right)$, one obtains

$$
\alpha=1-\Sigma_{a}\left(T_{1}\right), \quad \beta=K_{1}\left(T_{1}\right)
$$

Inserting the above result into Eq. (19), one obtains for $r \geq T_{2}$ the result

$$
\psi_{2}(r)=\left(1-\Sigma_{c}\left(T_{1}\right)\right) \sin (k r)+\left(K_{1}\left(T_{1}\right)+\Sigma_{s}\left(T_{2}\right)\right) \cos (k r) .
$$

The first order perturbation result for the above expressions is obtained by replacing $\psi_{2}$ by $\psi_{1}$ in the integrals for $\Sigma_{c}$ and $\Sigma_{s}$ in Eqs.above. Denoting the corresponding integrals from $T_{1}$ to $T_{2}$ by $I_{c}$ and $I_{s}$, respectively

$$
\begin{aligned}
& I_{c}=-\frac{1}{k} \int_{T_{1}}^{T_{2}} \cos \left(k r^{\prime}\right) V^{\prime}\left(r^{\prime}\right) \psi_{1}\left(r^{\prime}\right) d r^{\prime} \\
& I_{s}=-\frac{1}{k} \int_{T_{1}}^{T_{2}} \sin \left(k r^{\prime}\right) V^{\prime}\left(r^{\prime}\right) \psi_{1}\left(r^{\prime}\right) d r^{\prime},
\end{aligned}
$$

one finally obtains the first order result for $K_{1}\left(T_{2}\right)$

$$
K_{1}^{(1)}\left(T_{2}\right)=\frac{K_{1}\left(T_{1}\right)+I_{s}}{1-I_{c}} \simeq K_{1}\left(T_{1}\right)+I_{s}+K_{1}\left(T_{1}\right) \times I_{c} .
$$

This is our final expression. The numerical evaluation was carried out initially by using MATHEMATICA 19 to evaluate the integrals involving products of circular functions and $1 / r^{6}$. For example, if $T_{1}$ and $T_{2}$ are set equal to 500 and $2000 a_{0}$ respectively, and using for $C_{6}$ the value stated above, one finds $I_{c}=-8.443 \times 10^{-5}$ and $I_{s}=-1.6105 \times 10^{-5}$. Using for $K_{2}(500)$ the value from Table 3, one obtains from Eq. (26) the result

$$
K_{2}^{(1)}(2000)-K_{2}(500)=1.0265 \times 10^{-5},
$$

which compares very well with the numerical result from Table 3

$$
K_{2}(2000)-K_{2}(500)=1.0264 \times 10^{-5}
$$

The results for the scattering length and the effective range, described in the text, were calculated by a FORTRAN code which expresses the integrals $\int_{x}^{\infty}[\sin (t) / t] d t$ and $\int_{x}^{\infty}[\cos (t) / t] d t$ in terms of the functions $\mathrm{Ci}$ and Si. The latter were called from the IMSL scientific library, and generalization to larger powers of $t$ in the denominator (like $t^{6}$ in the present case) were obtained recursively through integrations by part. 


\section{B. The negative energy channel.}

For the negative energy channel a similar perturbative procedure will now be described. In what follows $\psi_{1}$ and $\psi_{2}$ now refer to the negative energy channel but otherwise they have the same meaning. The function $\psi_{1}$ coincides with the numerical solution $\psi_{N}$ for $r \leq T_{1}$, while $\psi_{2}$ for $T_{1} \leq r$ is given by

$$
\psi_{2}(r)=\gamma G_{2}(r)-\frac{1}{\kappa} F_{2}(r) \int_{r}^{T_{2}} G_{2} V^{\prime} \psi_{2} d r^{\prime}-\frac{1}{\kappa} G_{2}(r) \int_{T_{1}}^{r} F_{2} V^{\prime} \psi_{2} d r^{\prime} .
$$

Here $F_{2}(r)$ and $G_{2}(r)$ are equal, respectively to $\exp (-\kappa r)$ and $\sinh (\kappa r)$. By setting $\psi_{2}$ equal to $\psi_{1}$ at $r=T_{1}$ one obtains

$$
\gamma=\frac{1}{G_{2}\left(T_{1}\right)}\left(\psi_{1}\left(T_{1}\right)-\frac{1}{\kappa} \int_{T_{1}}^{T_{2}} G_{2}\left(r^{\prime}\right) V^{\prime}\left(r^{\prime}\right) \psi_{2}\left(r^{\prime}\right) d r^{\prime} F_{2}\left(T_{1}\right)\right),
$$

Inserting the above into Eq.(27) evaluated at $r=T_{2}$, replacing $\psi_{2}$ in the integrals in the above expressions by

$$
\psi_{2}(r) \simeq K_{2}\left(T_{1}\right) G_{2}(r)
$$

and dividing the result by $G_{2}\left(T_{2}\right)$, one obtains a preliminary value for $K_{2}\left(T_{2}\right)$

$$
\bar{K}_{2}\left(T_{2}\right)=K_{2}\left(T_{1}\right)-\frac{F_{2}\left(T_{1}\right)}{G_{2}\left(T_{1}\right)} I_{e}+I_{h s}
$$

where

$$
\begin{gathered}
I_{e}=-\frac{K_{2}\left(T_{1}\right)}{\kappa} \int_{T_{1}}^{T_{2}} G_{2}\left(r^{\prime}\right) V^{\prime}\left(r^{\prime}\right) G_{2}\left(r^{\prime}\right) d r^{\prime} \\
I_{h s}=-\frac{K_{2}\left(T_{1}\right)}{\kappa} \int_{T_{1}}^{T_{2}} F_{2}\left(r^{\prime}\right) V^{\prime}\left(r^{\prime}\right) G_{2}\left(r^{\prime}\right) d r^{\prime}
\end{gathered}
$$

The final value of $K_{2}\left(T_{2}\right)$ is obtained by dividing $\bar{K}_{2}\left(T_{2}\right)$ by the same normalizing factor $\left(1-I_{c}\right)$ which was required to normalize the wave function in channel 1 so that the coefficient in front of $\sin (k r)$ be equal to 1 . The final expression is

$$
K_{2}\left(T_{2}\right)=\frac{\bar{K}_{2}\left(T_{2}\right)}{\left(1-I_{c}\right)} \simeq K_{2}\left(T_{1}\right)\left(1+I_{c}\right)-\frac{F_{2}\left(T_{1}\right)}{G_{2}\left(T_{1}\right)} I_{e}+I_{h s}
$$

Using for $T_{1}$ and $T_{2}$ the values $500 a_{0}$ and $2000 a_{0}$ respectively, numerical evaluation of Eq. (29) gives $K_{2}\left(T_{2}\right)-K_{2}\left(T_{1}\right) \simeq-5.445 \times 10^{-4}$ while the numerical value obtained from Table 3 gives $\simeq-5.430 \times 10^{-4}$ 


\section{References}

${ }^{1}$ J. Weiner, V. Bagnato, S Zilio and P. S. Julienne, Rev. Mod. Phys. 71, 1 (1999);

${ }^{2}$ A. Fioretti, D. Comparat, C. Drag, T. F. Gallagher, and P. Pillet, Phys. Rev. Lett. 82, 1839 (1999).

${ }^{3}$ R. G. Gordon, J. Chem. Phys. 51, 14 (1969), R. G. Gordon, in Methods in Computational Physics ,( B. J. Alder, S. Fernbach and M. Rotenberg, Eds.), Vol. 10, pp 81-110. Academic Press, New York, 1971);

${ }^{4}$ Gh. Adam, L. Gr. Ixaru and A. Corciovei, J. Comput. Phys. 22, 1-33 (1976) and references therein.

${ }^{5}$ J. P. Burke Jr., C. H. Greene, and B. D. Esry, Phys. Rev. A54, 3225, (1996); K. J. Bathe, Finite Element Procedures in Engineering Analysis, Prentice Hall, Englewood Cliffs, NJ, 1976; K. J.Bathe and E. Wilson, Numerical Methods in Finite Element Analysis, Prentice Hall, Englewood Cliffs, NJ, 1976; L. R. Ram-Mohan, S. Saigal, D. Dossa, and J. Shertzer, Comput. Phys. 4, 50 (1990).

${ }^{6}$ R. A. Gonzales, J. Eisert, I. Koltracht, M. Neumann and G. Rawitscher, J. of Comput. Phys. 134, 134 (1997);

${ }^{7}$ R. A. Gonzales, S.-Y. Kang, I. Koltracht, and G. Rawitscher, Integral Equation Method for Coupled Schrödinger Equations, J. of Comput. Phys., J. Comput. Phys. 153, 160 (1999);

${ }^{8}$ T. E. Simos and Ch. Tsitouras, J. Comput. Phys. 130, 123 (1997); A.D. Raptis and J. R. Cash, Comp. Phys. Commun. 44,95 (1987); T. E. Simos, Accurate Computations for the elastic scattering phase-shift problem, Computers Chem. 21, 125 (1997), and references there in.

${ }^{9}$ R. Kosloff, J. Phys. Chem. 92, 2087 (1988);

${ }^{10}$ E. Fattal, R. Baer and R. Kosloff, Phys. Rev. E 53, 1217 (1966);

${ }^{11}$ V. Kokoouline, O. Dulieu, R. Kosloff and F. Masnou-Seeuws, J. Chem. Phys., 110, 9865 (1999);

${ }^{12}$ E. R. Bittner and J. C. Light, J. Chem. Phys. 102, 2614 (1995);

${ }^{13}$ M. Marinescu, H. R. Sadeghpour and A. Delgarno, Phys. Rev. A 49, 892 (1994).

${ }^{14}$ Chris H. Greene, Phys. Rev. A 28, 2209 (1983); H. Le Rouzo and G. Raseev, Phys. Rev. A 29, 1214 (1984); M. Aymar, Chris H. Greene, and E. LucKoenig, Revs. Mod. Phys. 68,1016 (1996). 
${ }^{15}$ E. P. Wigner, Phys. Rev. 70, 15 (1946); E. P. Wigner and L. Eisenbud, Phys. Rev. 72, 29 (1947); T Teichman and E. P. Wigner, Phys. Rev. 87, 123 (1952).

${ }^{16}$ E. Anderson, Z. Bai, C. Bischof, J. Demmel, J. Dongarra, J. Du Cros, A. Greenbaum, S. Hammerling, A. Mckenney, S. Ostouchov, and D. Sorensen, LAPACK Users Guide, (SIAM, Philadelphia, 1992).

${ }^{17}$ F. H. Mies, Phys. Rev. A 7, 957 (1973).

${ }^{18}$ J. P. Burke, Jr., C. H. Greene, and J. L. Bohn, Phys. Rev. Lett. 81, 3355 (1999); Bo Gao, Phys. Rev. A58, 4222 (1998), ibid 58, 1728 (1998)

${ }^{19} \mathrm{~S}$. Wolfram Mathematica: A System for doing Mathematics by Computer, Second Edition. Addison-Wesley, Redwood City, CA, 1991. 


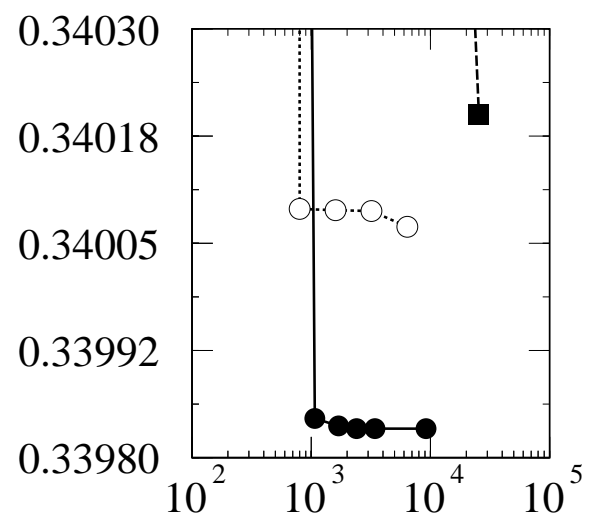

Number of mesh points 


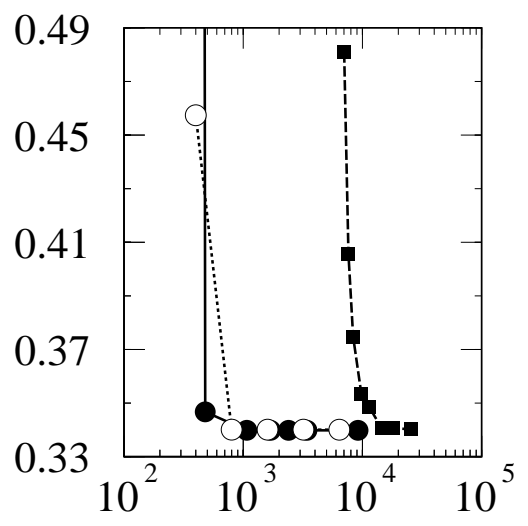

Number of mesh points 


\section{Cold collision}

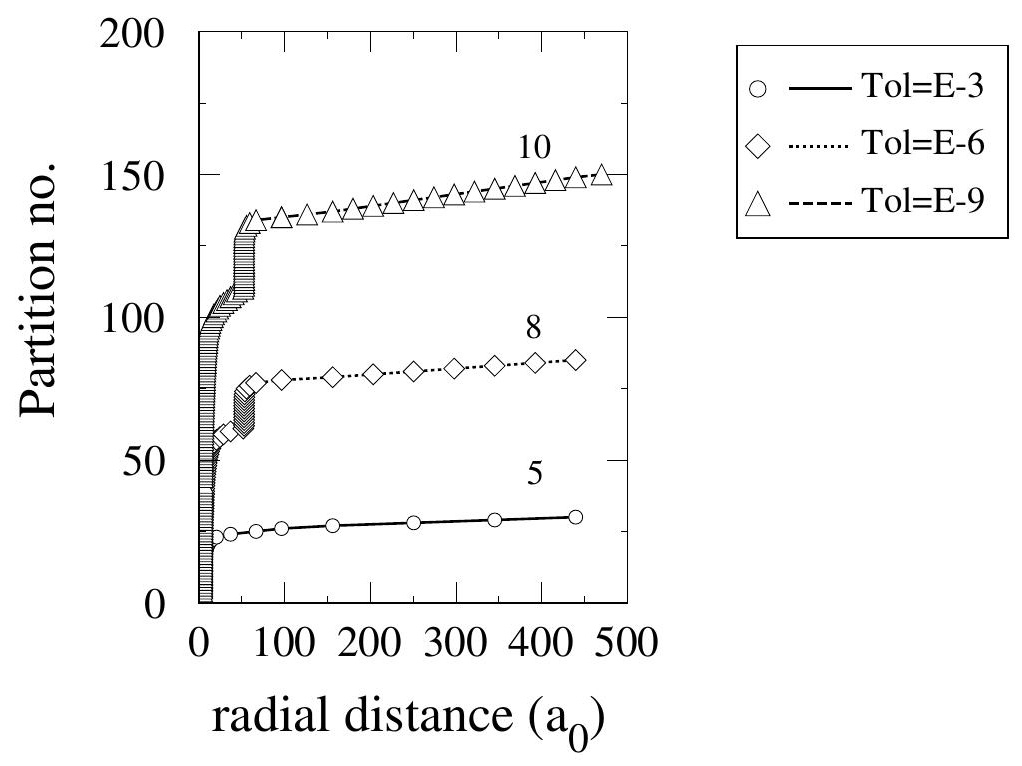

